\title{
Effect of Strain on Viscoelastic Behavior of PVP-CMC Based Medicated Hydrogels
}

\author{
Niladri Roy ${ }^{\mathrm{a}}$, Nabanita Saha ${ }^{\mathrm{b}}$, Takeshi Kitano ${ }^{\mathrm{b}}$ and Petr Saha ${ }^{\mathrm{b}}$ \\ ${ }^{a}$ Centre of Polymer Systems, Tomas Bata University in Zlin, \\ nam. T. G. Masaryka 5555, 76001 Zlin, Czech Republic. \\ ${ }^{b}$ Centre of Polymer Systems, Polymer Centre, Tomas Bata University in Zlin, \\ nam. T. G. Masaryka 5555, 76001 Zlin, Czech Republic.
}

\begin{abstract}
PVP-CMC and PVP-CMC-BA are designated as medicated hydrogels which are elastic in nature like other gels and solids. This present work was focused on the effect of strain on viscoelastic behavior of these medicated hydrogels. Dynamic frequency sweep test at $10 \%$ strain and dynamic strain sweep tests at different angular frequencies were performed on these hydrogels. Freshly prepared hydrogels and hydrogels swelled for $60 \mathrm{~min}$ from dry state were chosen for the experiments. These two experiments reveal that both PVP-CMC and PVP-CMC-BA hydrogels (fresh and swelled) maintain elastic behavior from very low to medium strain, but at high strain they become viscous in nature.
\end{abstract}

Keywords: Hydrogels, Frequency, Strain, Swelling, Viscoelastic.

PACS: $83.85 . \mathrm{Cg}, 83.85 . \mathrm{Vb}, 87.85 . \mathrm{J}, 87.16 . \mathrm{dm}$

\section{INTRODUCTION}

The medicated hydrogels are nothing but a giant structure made of polymer molecules that are branched in three dimensions and forms an inter-connected network spread out through the entire medium as a dispersed phase with the continuous phase being the water, and they are non-toxic and biocompatible in nature [1]. Due to their high water content and rubbery consistency, similar to natural tissue, as well as their biocompatibility, hydrogels derived from synthetic or natural materials are used in clinical and experimental medicine for a wide range of applications, like contact lenses, implants, controlled release systems, electrode gels, wound dressings, coating and controlled water permeable membranes [2]. Wound healing is a dynamic process and a dressing can enhance the progress of healing. Motivated by these interesting features of hydrogels and its demand in health care purposes we prepared a simple technology oriented biopolymer based novel medicated hydrogels which is human friendly, biocompatible, medicinal value-added, moreover appropriate to be used as dressing materials [3-5]. Many different kinds of hydrogels and their efficiency in biomedical application have been reported through the years [6-9]. Among the other properties, characterization of the rheological properties of soft materials like hydrogel is important [10], because these materials are viscoelastic, they not only store elastic energy when deformed, but also dissipate energy like a viscous fluid [11]. The use of 
oscillatory rheological analysis in investigating the mechanical properties of hydrogels is well known and continues to be one of the techniques most commonly used in hydrogel characterization [8]. There has been an accumulation of evidences that hydrogel networks exhibit highly enhanced viscoelastic properties which are quite different from ordinary polymer melts. Viscoelastic measurements revealed the following characteristics: the glass transition temperature is increased due to the reduced mobility of a polymer chain as a result of the physical crosslinking; hence, the rubbery plateau is extended [12]. The rheological property measurement under a wide range of deformation and its rate is important for wound dressing hydrogels to know their flow behavior, which signifies their flexibility to apply on a wound, which is an uneven surface and always in a motion. Therefore, in this present paper we have focussed on the effect of strain on the viscoelastic behavior of hydrogels under oscillatory flow.

\section{EXPERIMENTAL}

PVP-CMC and PVP-CMC-BA hydrogels were prepared using moist heat treatment using polyvinylpyrrolidone (PVP), carboxymethyl cellulose (CMC), polyethylene glycol (PEG), agar, and glycerin, without or with boric acid (BA), respectively [3-5]. The composition of PVP-CMC and PVP-CMC-BA are depicted in Table 1. The experiments were carried out using the freshly prepared and swelled hydrogels (the dry hydrogel films were swollen for $60 \mathrm{~min}$ in distilled water), and designated as "fresh" and "swelled" hydrogels respectively for both PVP-CMC and PVP-CMC-BA. In our previous articles we had established that after 60 min of swelling from dry state, the hydrogels regain quite similar properties with the fresh hydrogels [3, 4]. The optical view of the hydrogels (fresh, dry and swelled) is shown in Figure 1.

TABLE 1. Composition of hydrogels (W/V\%).

\begin{tabular}{lccccccc}
\hline Sample Index & PVP & CMC & PEG & Agar & Glycerin & BA & Water \\
\hline PVP-CMC & 0.2 & 0.8 & 1 & 2 & 1 & 0 & 95 \\
PVP-CMC-BA & 0.2 & 0.8 & 1 & 2 & 1 & 3 & 92 \\
\hline
\end{tabular}

The viscoelastic behavior of hydrogels was investigated by using a parallel plate rheometer (ARES; Rheometrics Scientific, USA) testing machine with an "RSI Orchestrator" software package. A $25 \mathrm{~mm}$ diameter parallel plate measuring geometry, with a gap of about 2-3 mm was used, employing at strain amplitude (10\%), which is considered as higher strain than the limit for the measurements with the linear viscoelastic region (LVER). Dynamic frequency sweep tests were carried out at $28^{\circ} \mathrm{C}$ to observe the storage $\left(G^{\prime}\right)$ and loss $\left(G^{\prime \prime}\right)$ moduli, and complex viscosity $\left(\eta^{*}\right)$ as a function of a wide range of angular frequencies $\left(\omega: 0.1-100 \mathrm{rad} . \mathrm{s}^{-1}\right)$. Furthermore, dynamic strain sweep tests were also carried out at $28^{\circ} \mathrm{C}$ between the strain region of $0.1-100 \%$ at three different angular frequencies $\left(\omega: 0.39,3.9\right.$ and $\left.39 \mathrm{rad}_{\mathrm{s}}{ }^{-1}\right)$. All experiments were carried out in triplicates on individual samples. 


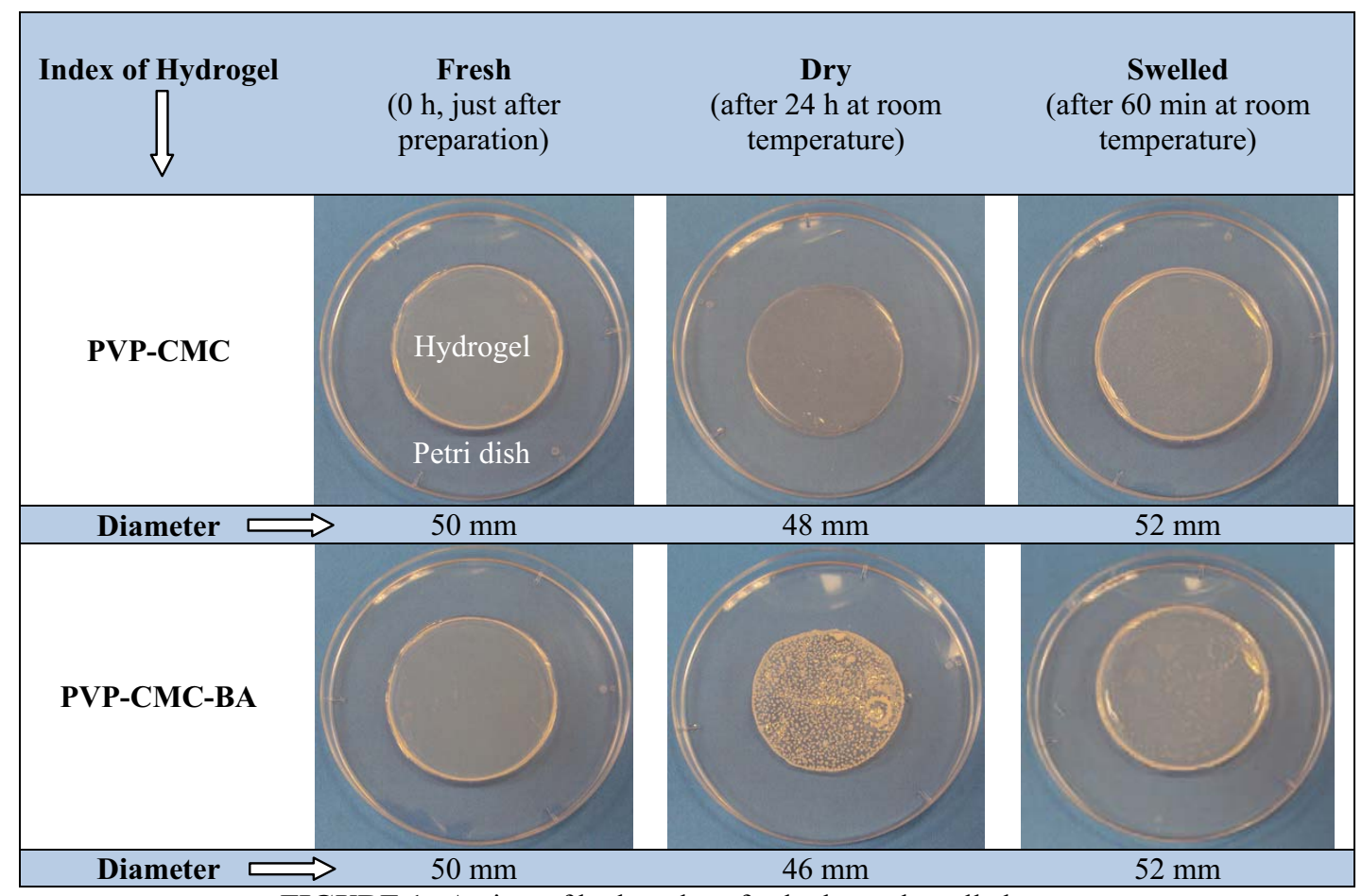

FIGURE 1. A view of hydrogels at fresh, dry and swelled state.

\section{RESULTS AND DISCUSSIONS}

\section{Dynamic Frequency Sweep Test}

Dynamic frequency sweep tests of PVP-CMC and PVP-CMC-BA hydrogels (fresh and 60 min swelled) were carried out at $10 \%$ strain amplitude to understand the effect of high strain on the viscoelastic properties of hydrogels. It is reported that at low strain (say 1\%), the hydrogels show rubbery consistency. The storage modulus $\left(G^{\prime}\right)$ values are much higher than loss modulus $(G$ ") values throughout, thus the elastic response dominates, which is typical for gels or solid like materials $[3,4,10]$. But the effect of high strain $(10 \%)$ on the rheological properties $\left(G^{\prime}, G^{\prime \prime}\right.$ and $\left.\eta^{*}\right)$ of hydrogels are shown in Figures 2-4, and the results seem significantly different from the low strain measurements. Loss modulus $\left(G^{\prime \prime}\right)$ values are always distinguishably higher than the storage modulus $\left(G^{\prime}\right)$ values during whole frequency range and the similar trends are observed in case of both fresh and swelled hydrogels. It represents that at higher strain the hydrogels lose their elastic nature and the viscous response dominates. Due to the high strain condition the physical bonding and entanglements, which are responsible for physical crosslinking in the hydrogels start to break, thus lose their elastic properties. In all cases the $G$ ' values for PVP-CMC-BA are higher than that of PVP-CMC up to a certain period, because incorporation of BA in hydrogel generates higher crosslinking density. Figure 5, as an evidence, illustrates the 
cross-sectional SEM view of freeze-dried hydrogel samples, and it is clear from the images that PVP-CMC-BA possesses denser network than PVP-CMC-BA. Figure 4 represents the effect of angular frequency $(\omega)$ at $10 \%$ strain on complex viscosity $\left(\eta^{*}\right)$ for the hydrogels (fresh and 60 min swelled), and it can be seen that $\eta^{*}$ values of the fresh hydrogels are higher than the swelled hydrogels. This is the effect of swelling, because during swelling some structural changes may occur within the hydrogel network.

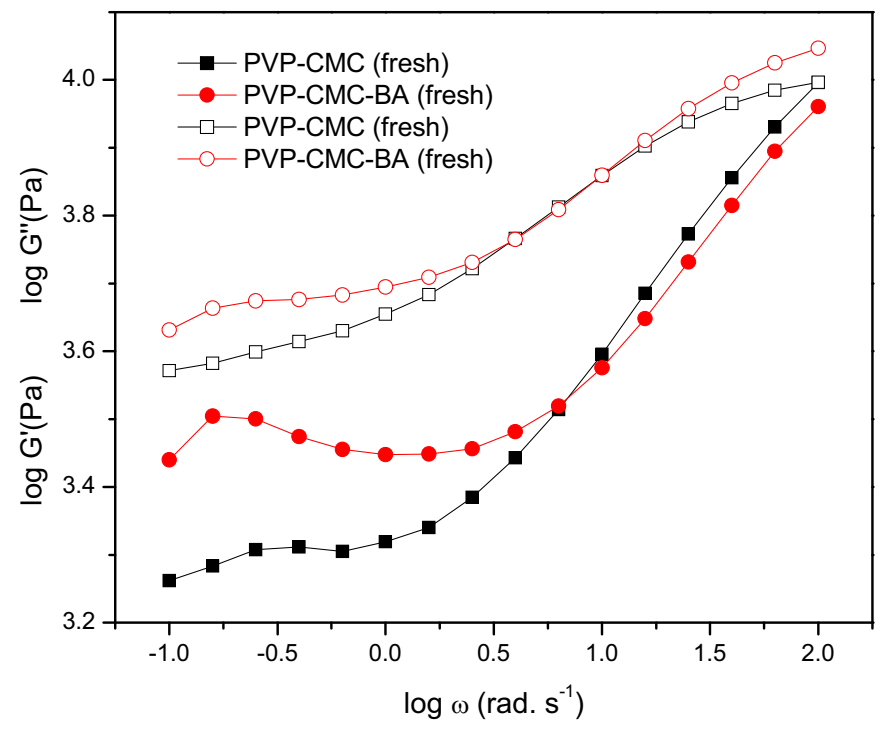

FIGURE 2. Effect of angular frequency $(\omega)$ at $10 \%$ strain on storage modulus ( $G$, filled symbols) and loss modulus ( $G$ ”, unfilled symbols) for the freshly prepared hydrogels.

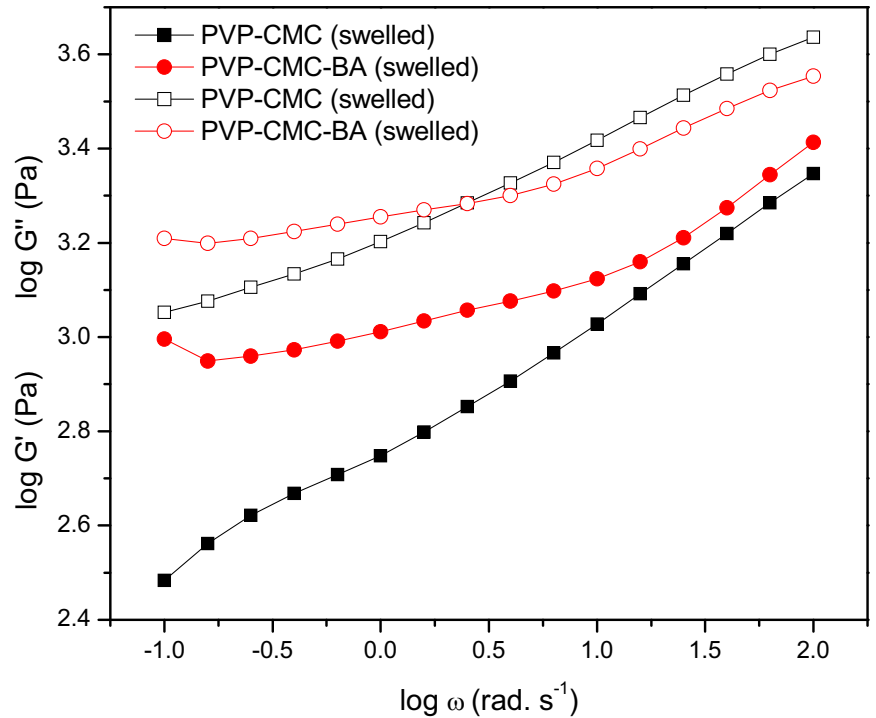

FIGURE 3. Effect of angular frequency $(\omega)$ at $10 \%$ strain on storage modulus ( $G$ ', filled symbols) and loss modulus ( $G$ ”, unfilled symbols) for the hydrogels after $60 \mathrm{~min}$ swelling. 


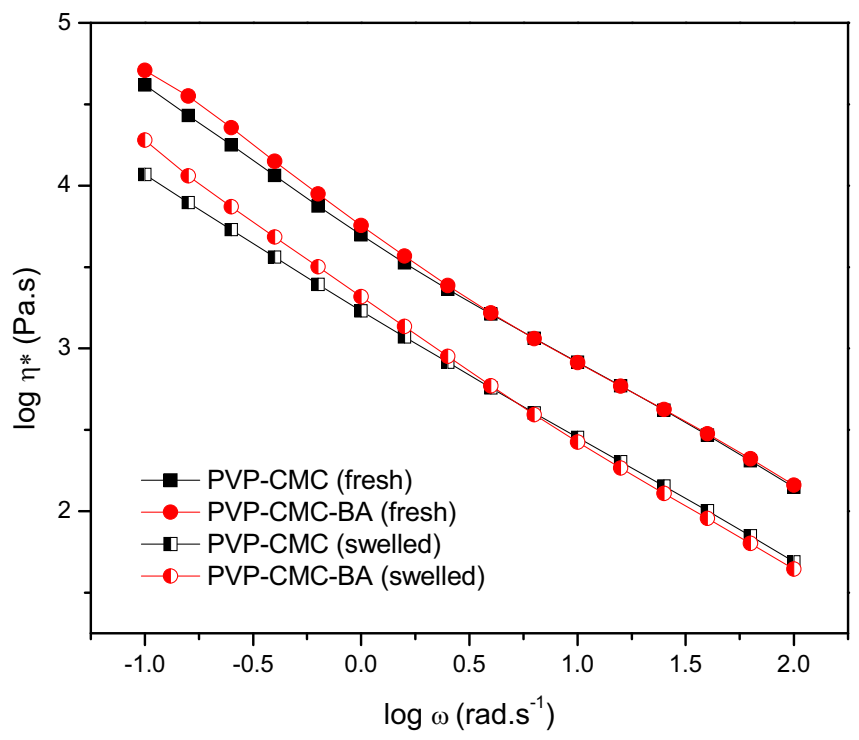

FIGURE 4. Effect of angular frequency $(\omega)$ at $10 \%$ strain on complex viscosity $\left(\eta^{*}\right)$ for the hydrogels (fresh and 60 min swelled).
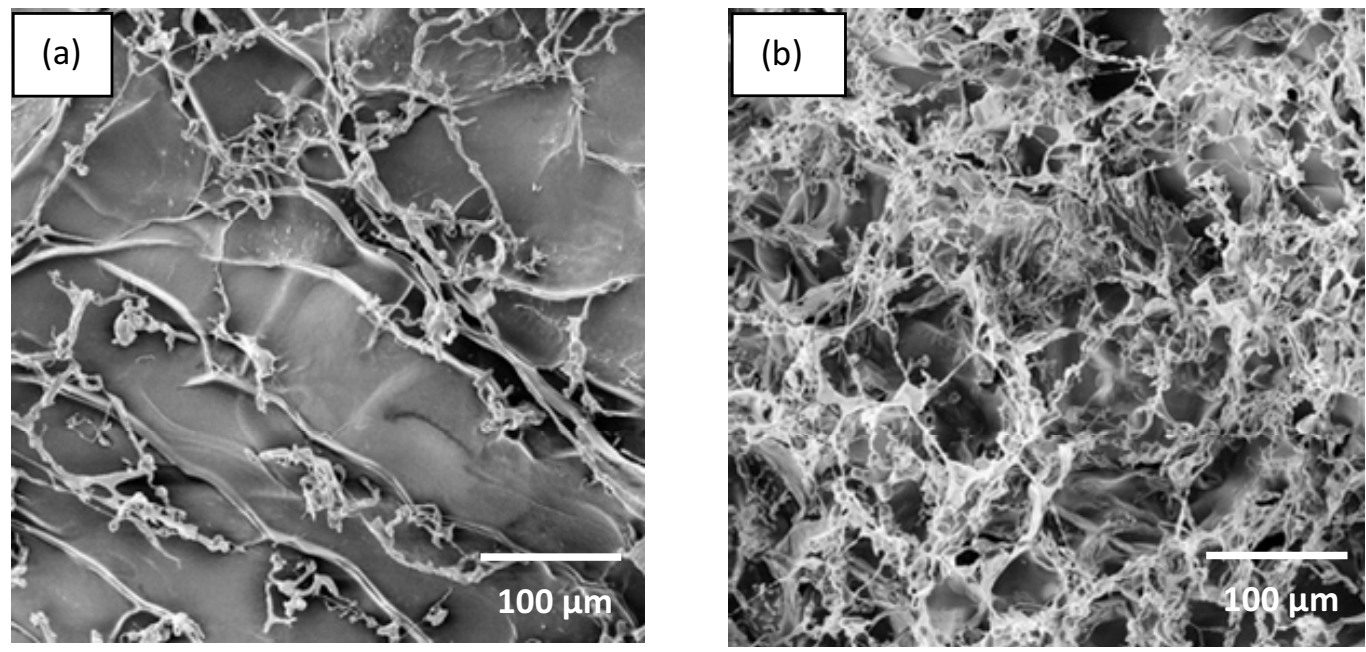

FIGURE 5. Cross-sectional SEM view of (a) PVP-CMC and (b) PVP-CMC-BA hydrogel. 


\section{Dynamic Strain Sweep Test}

The viscoelastic behavior of the PVP-CMC and PVP-CMC-BA hydrogels (fresh and swelled) was measured using dynamic strain sweep test between low to high strain region (0.1-100) at lower $\left(0.39 \mathrm{rad} . \mathrm{s}^{-1}\right)$, medium $\left(3.9 \mathrm{rad} . \mathrm{s}^{-1}\right)$ and higher $\left(39 \mathrm{rad} . \mathrm{s}^{-1}\right)$ angular frequencies, and the results are depicted in Figures 6 and 7.
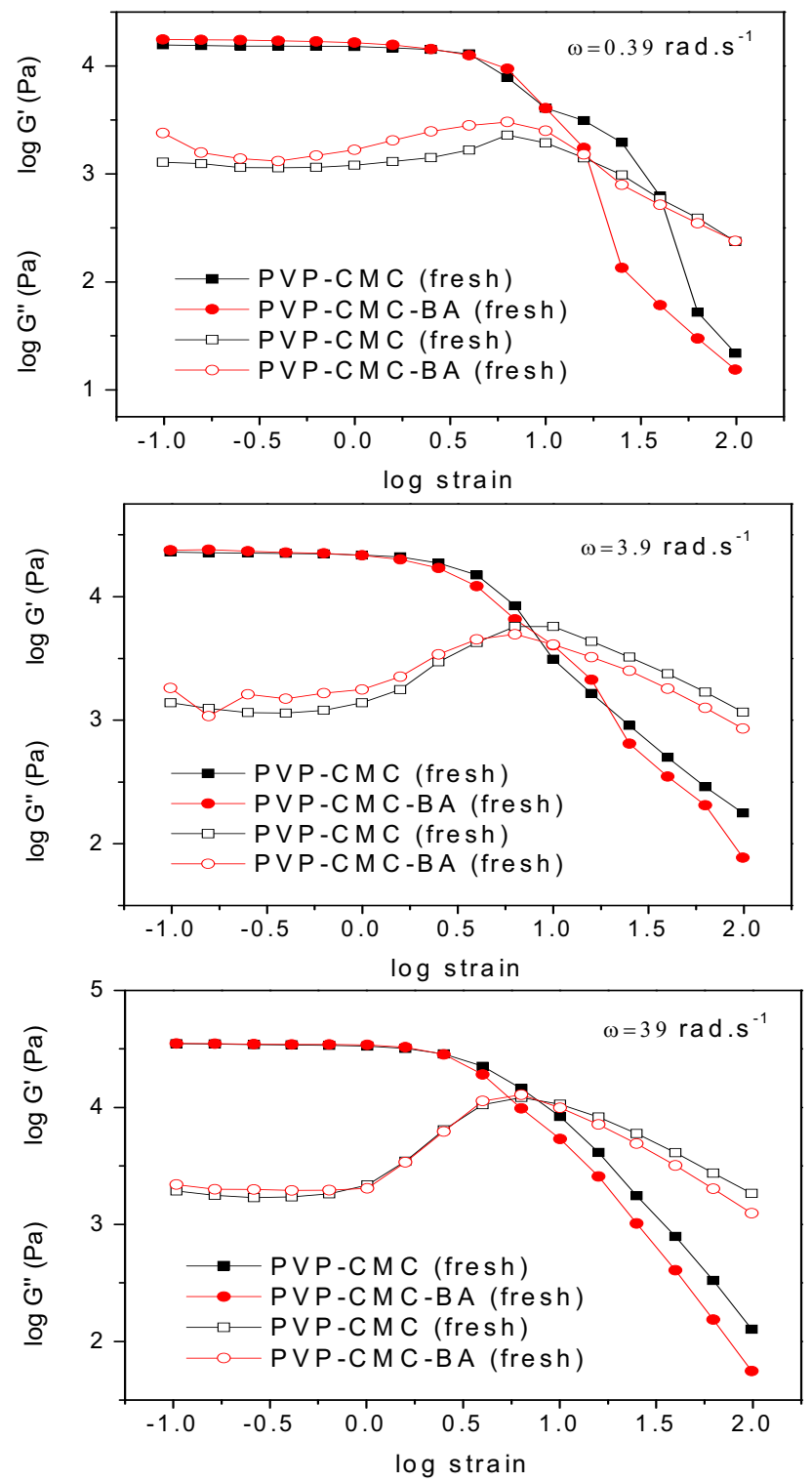

FIGURE 6. Effect of strain at different angular frequencies $(\omega)$ on storage modulus ( $G^{\prime}$, filled symbols) and loss modulus ( $G$ ", unfilled symbols) for the freshly prepared hydrogels. 

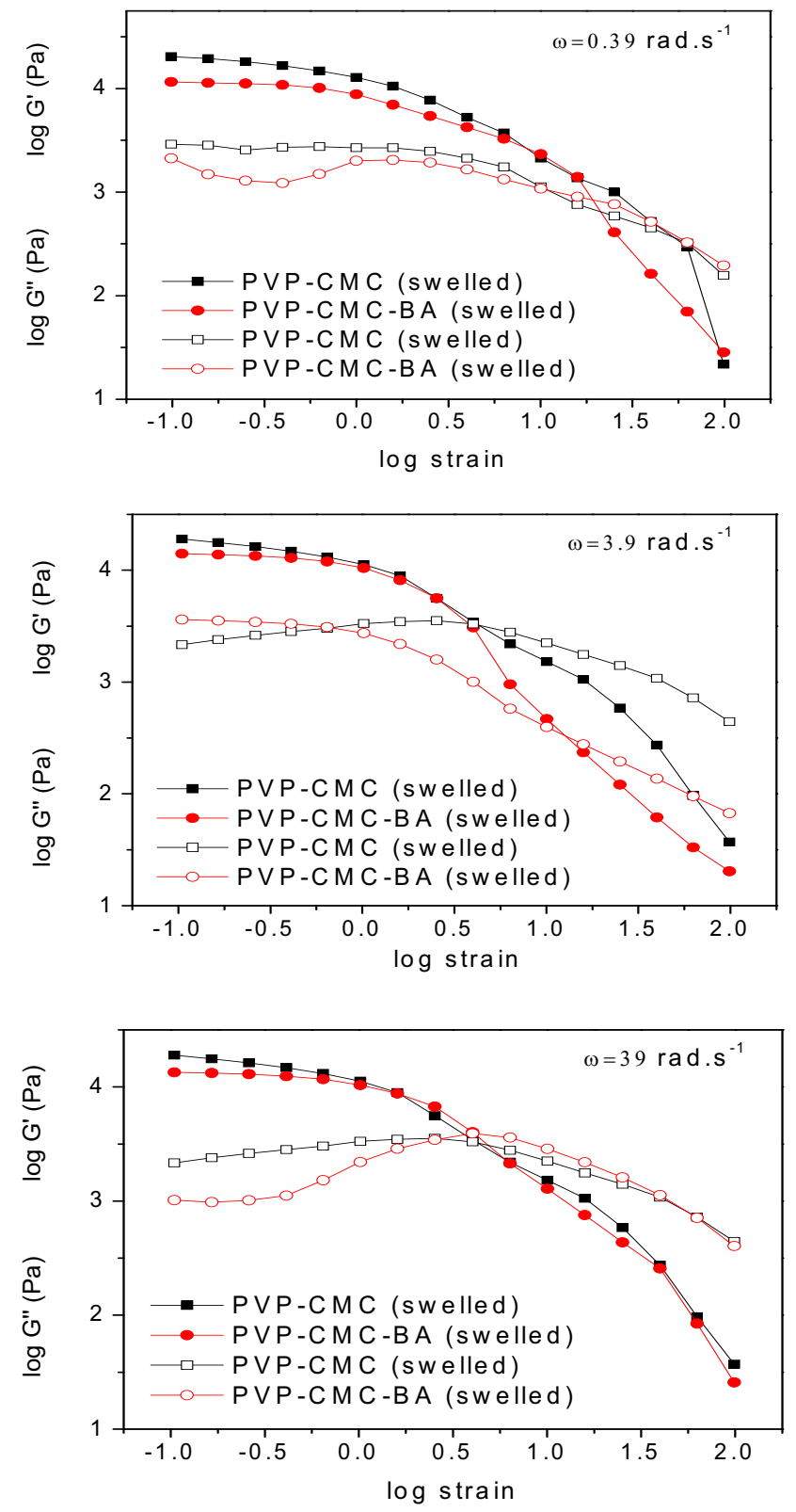

FIGURE 7. Effect of strain at different angular frequencies $(\omega)$ on storage modulus $\left(G^{\prime}\right.$, filled symbols) and loss modulus ( $G$,', unfilled symbols) for the 60 min swelled hydrogels.

It can be seen from Figures 6 and 7 that at the range of lower strain to medium strain regions the hydrogels more or less maintain their rubbery nature, i.e. their elastic properties, but after that with increase in strain the elastic property starts to decrease sharply and viscous property prevails. These behavior are coincident with the results shown in Figures 2 and 3, and can be explained in this way: up to a certain limit of strain the strength of the crosslinking networks in the hydrogel opposes the external force and maintain an equilibrium. But when the strain becomes very high, the bonds 
and networks in the hydrogels start to break or deformed, thus the hydrogels lose elasticity, and they become viscous predominant materials.

\section{CONCLUSION}

The dynamic frequency sweep test and dynamic strain sweep test revealed that, both PVP-CMC and PVP-CMC-BA hydrogels are elastic in nature when no or low strain is applied. However, with increase in strain, their viscoelastic properties show gradual change from elastic to viscous, and at high strain they turn into totally viscous.

\section{ACKNOWLEDGMENTS}

The authors are thankful to the Ministry of Education, Youth and Sports of the Czech Republic (MSM 70088352101) for financial support. This article was also created with partial support of Operational Programme Research and Development for Innovation co-funded by the European Regional Development Fund (ERDF) and national budget of Czech Republic within the framework of the Centre of Polymer Systems project (reg. number: CZ.1.05/2.1.00/03.0111).

\section{REFERENCES}

1. S. Chatterjee and H. B. Bohidar, J. Surface Sci. Technol. 22, 1-13 (2006).

2. D. B. Stein, Handbook of Hydrogels properties, Preparation and Application, New York: Nova Science Publishers, 2009, pp. ix.

3. N. Roy, N. Saha, T. Kitano and P. Saha, J. Appl. Polym. Sci. 117, 1703-1710 (2010).

4. N. Roy, N. Saha, T. Kitano and P. Saha, Soft Mater. 8, 130-148 (2010).

5. N. Roy, N. Saha, P. Humpolicek and P. Saha, Soft Mater. 8, 338-357 (2010).

6. J. Kopecek and J. Yang, Polym. Int. 56, 1078-1098 (2007).

7. O. Z. Higa, S. O. Rogero, L. D. B. Machado, M. B. Mathor and A. B. Lugao, Radiat. Phys. Chem. 55, 705-707 (1999).

8. J. G. Lyon, L. M. Geever, M. J. D. Nugent, J. E. Kennedy and C. L. Higginbotham, J. Mech. Behav. Biomed. Matter. 2, 485-493 (2009).

9. C. Yang, L. Xu, Y. Zhou, X. Zhang, X. Huang, M. Wang, Y. Han, Z. Wei, S. Zhai And J. Li, Carbohydr. Polym. 82, 1297-1305 (2010).

10. N. Roy, N. Saha, T. Kitano and P. Saha, AIP Conference Proceedings 1152, 210-216 (2009).

11. N. Yang, J. R. D. B. Bruyn and J. L. Hutter, La Physique au Canada 64, 141-143 (2008).

12. F. Tanaka and S. F. Edwards, Macromolecules 25, 1516-1523 (1992) 
Copyright of AIP Conference Proceedings is the property of American Institute of Physics and its content may not be copied or emailed to multiple sites or posted to a listserv without the copyright holder's express written permission. However, users may print, download, or email articles for individual use. 